\title{
STABILITY THEORY AND HAMILTONIAN DYNAMICS IN THE EULER IDEAL FLUID EQUATIONS
}

\author{
JOACHIM WORTHINGTON
}

(Received 6 August 2017; first published online 28 September 2017)

2010 Mathematics subject classification: primary 76E05; secondary 76B15, 76E09, 37K45.

Keywords and phrases: ideal fluids, shear flows, stability theory.

The study of shear flow steady states has led to a wealth of research in the field of fluid dynamics. By studying shear flows, we can understand how a fluid behaves and how coherent structures arise. In this thesis, we primarily study the stability of shear flows of the Euler equations. The Euler equations describe the dynamics of an ideal fluid which is incompressible, inviscid and experiences no external forces. These equations can be formulated as a Poisson system, which we exploit to study stability.

We study the family of shear flows of the Euler equations with vorticity of the form $\Omega(x, y)=\cos \left(\kappa_{x} p_{x} x+\kappa_{y} p_{y} y\right)$ on a two-dimensional periodic domain of size $\left[0,2 \pi / \kappa_{x}\right) \times\left[0,2 \pi / \kappa_{y}\right)$. We prove that if $p_{y}=0$ and $\kappa_{x}\left|p_{x}\right|<\kappa_{y}$, this shear flow is linearly stable. If $\sqrt{\kappa_{x}^{2} p_{x}^{2}+\kappa_{y}^{2} p_{y}^{2}}>(3+2 \sqrt{3}) / 2$, we prove that the corresponding shear flow is nonlinearly unstable. In addition, we discuss the spectrum of the linearisation of shear flows more generally and explore a related Jacobi problem. There is a known Poisson structure preserving truncation of the Euler equations. We prove analogous stability results in this system and discuss the qualitative differences. We extend a previously known Poisson integrator for this truncation of the Euler equations to a general two-dimensional periodic domain. Some of these results have appeared in [1] and [2].

The Euler equations on a three-dimensional periodic domain are less well understood. In this domain we formulate the dynamics in terms of the vorticity Fourier modes. This is then used to study shear flows and prove similar stability results as for the two-dimensional case. We show that the linearised equations split into subsystems which have equivalent dynamics to the subsystems of the two-dimensional linearised equations. This is used to prove the existence of a family of linearly stable shear

Thesis submitted to the University of Sydney in March 2017; degree awarded on 22 May 2017; primary supervisor Holger Dullin, associate supervisor Robert Marangell.

(c) 2017 Australian Mathematical Publishing Association Inc. 0004-9727/2017 \$16.00 
flows and another family of linearly unstable shear flows. For a dense set of parameter values, the linearised system has a nontrivial nilpotent part leading to linear instability. This is linked to the nonnormality of the system, which indicates a transition to turbulence. We also show that the Euler equations on a three-dimensional periodic domain can be formulated as a Poisson system. We finally present some numerical results demonstrating and exploring the results of this thesis.

\section{References}

[1] H. R. Dullin, R. Marangell and J. Worthington, 'Instability of equilibria for the two-dimensional Euler equations on the torus', SIAM J. Appl. Math. 76(4) (2016), 1446-1470.

[2] H. R. Dullin and J. Worthington, 'Stability results for idealized shear flows on a rectangular periodic domain', J. Math. Fluid Mech. (2017), doi:10.1007/s00021-017-0329-2.

JOACHIM WORTHINGTON, School of Mathematics and Statistics,

University of Sydney, NSW 2006, Australia

e-mail: joachim.worthington@gmail.com 